AOP Periodontology

\title{
Periodontal status of adults with systemic sclerosis: case- control study
}

\begin{tabular}{|r|l|}
\hline Journal: & Journal of Periodontology \\
\hline Manuscript ID: & JOP-10-0593.R1 \\
\hline Manuscript Type: & Original Article \\
\hline Author: & n/a \\
\hline Complete List of Authors: & $\begin{array}{l}\text { Leung, W. Keung; The University of Hong Kong, Faculty of Dentistry } \\
\text { Chu, Chun Hung; The University of Hong Kong, Faculty of Dentistry } \\
\text { Mok, Mo Yin; The University of Hong Kong, LKS Faculty of Medicine, } \\
\text { Department of Medicine } \\
\text { Yeung, K. W. Shadow; The University of Hong Kong, Faculty of } \\
\text { Dentistry } \\
\text { Ng, Sam K. S.; Hospital Authority, New Territories East Cluster of } \\
\text { Hospitals }\end{array}$ \\
\hline Key Words: & Oral medicine, Hospital dentistry, Internal medicine \\
\hline
\end{tabular}




\section{Periodontal status of adults with systemic sclerosis: case-control study}

W. Keung Leung*, Chun Hung Chu*, Mo Yin $\mathrm{Mok}^{\dagger}$, K. W. Shadow Yeung*, Sam K. S. $\mathrm{Ng}^{\ddagger}$

*Faculty of Dentistry, The University of Hong Kong, Hong Kong SAR, China

${ }^{\dagger}$ Li Ka Shing Faculty of Medicine, The University of Hong Kong, Hong Kong SAR, China

${ }^{\ddagger}$ New Territories East Cluster of Hospitals, Hospital Authority, Hong Kong SAR, China

Running title: Systemic sclerosis: periodontal status

The authors report no conflict of interest. This project was supported by the University of Hong Kong Research and Conference Grant (200807176127) and was partially supported by a grant from the Research Grants Council of the Hong Kong Special Administrative Region, China (772110M).

Key findings:

Hong Kong Chinese adults with systemic sclerosis seemed to exhibit higher levels of periodontal inflammation and wider radiographic periodontal ligament spaces than age- and sex- matched controls despite experiencing similar level of inadequate plaque control when compared to unaffected individuals. 


\section{Word counts}

Abstract: 231;

Body (excluding abstract, references, and figure legends): 2347.

Figures: 0; tables: 3.

Number of references: 26.

Date of resubmission: December 6, 2010 


\begin{abstract}
Background: Systemic sclerosis (SSc) is a systemic autoimmune disease that affects connective tissue in the skin, blood vessels and major organs of the body. This project aimed to compare the periodontal status of dentate Hong Kong Chinese with and without SSc.
\end{abstract}

Methods: Thirty-six non-smoking adults with SSc (35 women; $50.6 \pm 11.7$ years, free from Sjogren's syndrome) attending a teaching hospital were age- and sex-matched to systemically healthy controls attending a dental hospital. Both groups had similar demographic characteristics but a lower proportion of SSc patients were working or studying $(\mathrm{p}<0.05)$. Twenty-three $(32.0 \%)$ of all participants were regular dental attendees. Orthopantomogram rediograph were taken. Participants were surveyed and periodontally examined.

Results: Both groups had a similar number of erupted teeth, proportion of sites with detectable plaque, and mean full-mouth probing attachment level, whereas controls had less bleeding on probing $(\mathrm{BOP}(\%), 49.3 \pm 22.6 v s .78 .4 \pm 19.6 ; \mathrm{p}<0.001)$ and a shallower mean full-mouth probing pocket depth $(\operatorname{PPD}(\mathrm{mm}), 1.92 \pm 0.44$ vs. $2.52 \pm 0.58 ; \mathrm{p}<0.001)$. Radiographically, SSc patients had wider periodontal ligament spaces. (PDL (mm), $0.36 \pm$ 0.06 vs. $0.33 \pm 0.03, \mathrm{p}<0.01)$

Conclusion: Hong Kong Chinese SSc adults seem to exhibit higher levels of periodontal inflammation and wider radiographic PDL spaces than age- and sex- matched controls. Future studies are warranted to further investigate any associations between these 
periodontal features and SSc.

\section{KEY WORDS}

Case-control study; periodontal diseases; scleroderma, systemic 
Systemic sclerosis (SSc), or scleroderma, is a systemic autoimmune disease that affects connective tissue in the skin and underlying tissues, including blood vessels and major organs of the body. The pathology of SSc is likely to involve the activation of immune cells and subsequent fibrotic changes in the skin, internal organs, and small blood vessels. ${ }^{1}$ Two types of SSc, limited and diffuse, have been described. Patients with limited SSc may present with calcinosis, Raynaud's phenomenon, esophageal dysmotility, sclerodactyly, and telangiectasia, together known as CREST. Patients with diffuse SSc develop skin thickening over all parts of the body, including the trunk, and are more likely than patients with limited SSc to develop severe organ problems. Only a short interval elapses between the onset of Raynaud's phenomenon and significant organ involvement (Walker et al. 2007). ${ }^{2}$

The reported prevalence of SSc ranges from 4 to 286 per million and the incidence rate varies from 0.6 to 122 per million per year. ${ }^{3}$ The peak age of onset of SSc is between 30 and 50 years. ${ }^{4}$ The wide ranges of these figures may reflect differences in disease definition, susceptibility to SSc on a genetic basis, or exposure to putative environmental triggers. Nevertheless, a high female to male ratio is a consistent finding. SSc may also coexist with other autoimmune conditions. For example, simultaneous presentation of systemic lupus erythematosus or polymyositis, or both, with SSc is termed "mixed connective tissue disorder". In addition, up to $14 \%$ of SSc patients may have secondary 
Sjögren's syndrome, particularly those with limited cutaneous SSc. ${ }^{6}$

There is currently no effective treatment to control the overproduction of fibrotic tissue in individuals with SSc. Management approaches mainly focus on relieving symptoms and limiting organ damage. ${ }^{7}$ Anti-inflammatory drugs and immunosuppressants are often used to treat underlying rheumatological problems. ${ }^{8}$ Vasodilators, calcium channel blockers, and angiotensin converting enzyme inhibitors can control symptoms of visceral complications and Raynaud's phenomenon, ${ }^{6}$ while analgesics can control joint and muscle pain. ${ }^{7}$ Stretching exercises under the instruction of physiotherapists and occupational therapists can maintain joint mobility. ${ }^{7}$

A common oral complication in SSc is constriction of the oral orifice. ${ }^{6}$ Progressively limited mouth opening eventually leads to so-called "fish mouth". SSc is reported to be associated with an increased risk of dental caries, oral ulcers, and fungal infections, ${ }^{9}$ as well as loss of tongue mobility with fibrotic indurations, ${ }^{10}$ dry mouth (salivary hypofunction), and telangiectasia. ${ }^{11}$ Facial bones may sometimes become eroded, as has been reported in individual studies and case reports showing osseous resorption at the mandibular angles, coronoid processes, and zygomatic arches. ${ }^{12,13}$

Furthermore, SSc is associated with a high prevalence of periodontal disease. ${ }^{9}$ Supporting evidence includes radiological findings such as widening of periodontal ligament spaces, ${ }^{13-16}$ abnormalities in periodontal microcirculation observable using 
periodontal capillaroscopy, ${ }^{17}$ and loss of gingival attachment and prominent buccal mucosal crenations. ${ }^{10}$ However, a comprehensive report on the exact clinical periodontal status of SSc patients is lacking. The aim of this cross-sectional case-control study was therefore to compare the periodontal conditions of dentate adults with SSc and those of age- and sex-matched non-SSc controls.

\section{MATERIALS \& METHODS}

\section{Participants}

This convenience sample was drawn from 77 Chinese SSc patients (73 women, 4 men), free from Sjogren's syndrome, attending the Rheumatology Clinic of the Department of Medicine during 2008, at Queen Mary Hospital, a medical teaching hospital of The University of Hong Kong, serving approximately 40\% (0.6 million) of the population in western Hong Kong Island. Twenty-one patients could not participate in this study because they were either hospitalized or institutionalized. Of the 56 ambulatory SSc patients, 43 indicated their availability but 2 of them were edentulous while one was current smoker. These three cases were therefore excluded. At the end of the clinical examination period, a total of 36 dentate SSc patients (35 women and 1 man) were recruited, four did not attend the dental examination arranged. Their age ranged from 29 to 76 years (mean $50.6 \pm 11.7$ 
years); and 25 had limited SSc and 11 had diffuse SSc. In addition, 14 (38.9\%) were receiving prednisolone, 6 (16.7\%) azathioprine, 9 (25.0\%) hydroxychloroquine, $11(30.6 \%)$ penicillamine, and $7(19.4 \%)$ colchicines. Overall, the patients had had SSc for 12.3 years (SD, 9.8 years; range 1 - 37 years). None of the controls had any systemic diseases or were currently taking any drugs.

All eligible patients were invited by telephone to participate in this study, given the opportunity to ask questions and receive clarification about the project, and invited to attend an oral examination at the Prince Philip Dental Hospital (PPDH), the dental teaching hospital of the Faculty of Dentistry, the University of Hong Kong. Age- and sex-matched dentate, non-SSc controls who were systemically healthy were drawn prospectively from the newly admitted pool of Chinese patients at the Reception and Primary Care Clinic of the PPDH over 3 months after the recruitment of SSc patients had ended. All control subjects should be non-smoker, without any prior history of autoimmune condition or systemic disease and were under no concurrent medication.

\section{Diagnosis of SSc}

SSc was classified as limited or diffuse depending on the extent of skin involvement according to LeRoy and co-workers. ${ }^{18}$ Patients who had concurrent autoimmune conditions such as systemic lupus erythematosus, polymyositis, and Sjogren's syndrome 
were excluded from study. Disease duration was measured from the first onset of manifestations apart from Raynaud's phenomenon.

\section{Ethics}

The ethical principles defined by the World Medical Association Declaration of Helsinki were followed in this study. The Institutional Review Board of The University of Hong Kong approved the study (UW 08-305). Written informed consent was obtained from each participant before study admission.

\section{Examination}

On the day of the oral examination, SSc and control participants completed a questionnaire regarding demographic characteristics, medical history, dental attendance, and oral hygiene habits. The questionnaire was checked for completeness and content and any queries were clarified before the examination. The medical status of the SSc patients was confirmed from Queen Mary Hospital Rheumatology Clinic patient records. No attempt was made to mask the patient group from the examiner because the physical features of SSc are easily recognizable.

The oral examination was conducted at the PPDH Periodontology Clinic by WKL. 
The number of erupted teeth was recorded, and a periodontal examination was performed on six sites of each standing tooth (mesio-buccal, mid-buccal, disto-buccal, mesio-lingual, mid-lingual, disto-lingual) using a manual probe. ${ }^{19}$ The percentage of sites with detectable plaque $(\mathrm{P} 1 \%)$ and with bleeding on probing $(\mathrm{BOP} \%)$, and the probing pocket depth (PPD) and probing attachment level (PAL) were recorded. The periodontal ligament (PDL) widths around selected teeth in orthopantomogram ${ }^{* *}$ were studied as described by Alexandridis and White, ${ }^{16}$ except measurements were done using digitized image and computer software. The selected teeth were a maxillary central incisor, a mandibular lateral incisor, a mandibular canine, a mandibular first premolar, a maxillary second premolar, a maxillary first molar, and a mandibular second molar. The PDL widths were measured at one third and two thirds of the distance between the cemento-enamel junction and the apex of each tooth as located by a Schei ruler, both on the mesial and on the distal surface of each selected tooth.

The radiographs were scanned and digitized at a resolution of $1000 \mathrm{dpi}^{\dagger \dagger}$. An image processing program ${ }^{+}$was used to measure the width of the PDL space (in mm). Before each measurement, the system was calibrated with an optical meter (396 pixels/mm). The measurements were taken by one author (KWSY) who was trained in the measurement

\footnotetext{
${ }^{* *}$ Kodax Lanex regular extra-oral image screen plus panelisp films, taken with a Gendex Orthoralix SD Ceph machine, 70-72 kV, 9-10 mA, 10-12 seconds exposure, 1.23× magnification.

${ }^{\dagger \dagger}$ Perfection V700 Photo scanner with Dual Lens ${ }^{\mathrm{TM}}$ System; Epson, Suwa, Nagano, Japan.

I ImageJ, National Institutes of Health, Bethesda, Maryland, US.
} 
procedures, and was unaware of the patient grouping. Two independent measurements at each position were made at least a week apart and averaged.

\section{Statistical Analysis}

Data were analyzed using the statistical software package SPSS (16.02), and were initially tested for normality with the Kolmogorov-Smirnov test. The paired $t$-test was used to compare dental, periodontal, and radiographic data between SSc and control groups. Chisquare test was used to analyze categorical data among SSc and control groups. A predetermined chi-square probability of 0.05 or less, that is alpha level of significance (0.05), was set for rejecting the null hypothesis.

\section{RESULTS}

Although a lower proportion of SSc patients than of controls were working or studying, other demographic characteristics, dental attendance, and oral hygiene habits were generally similar (Table 1). Similar proportions of the SSc patients and controls $(27.8 \%$ and $36.1 \%$, respectively) had made their last dental visit within the previous year. The majority of both groups reported brushing their teeth twice daily.

There were no significant differences between control and SSc groups in the number 
of teeth remaining, P1\%, percentage of sites with a PPD of $6 \mathrm{~mm}$ or greater, and mean fullmouth PAL. However, SSc patients had a significantly higher BOP\%, mean full-mouth PPD, and percentage of sites with a PPD of 4 to $5 \mathrm{~mm}$ than the controls (Table 2).

As described by Alexandridis and White, ${ }^{16}$ maxillary central incisors, second premolars, first molars and mandibular lateral incisors, canines, first premolars, second molars were selected for radiographic analysis (Table 3). The SSc group appeared to have wider mean PDL spaces overall, and separately by anterior, posterior, upper, or lower position (Table 3).

\section{DISCUSSION}

One of the limitations of this study is the SSc people and the controls were not from the same sub-population of university general hospital attendees. However, this is very difficult to obtain in a clinical study like the current one. This case-control study has revealed some periodontal characteristics that are associated with SSc. Although the survey consisted of a convenience sample of ambulatory SSc patients without other concurrent autoimmune conditions attending a university hospital, the controls were ageand sex-matched, and also convenience sample of attendees at a university dental hospital. The groups were generally also matched by socio-demographic data, with the two groups 
reporting similar dental visit, and brushing habits. However, as the current sample size was small, care should be taken when interpreting association between SSc and poor oral health.

Both patient and control groups had inadequate oral hygiene and similar levels of a mean high level of periodontal destruction ${ }^{20}$ possibly reflective of the nature of the subjects recruitment protocol. ${ }^{19}$ However, interestingly, SSc patients experienced a higher BOP\% and deeper full-mouth PPD. The latter was probably due to a higher proportion of pockets of 4 to $5 \mathrm{~mm}$, and may have meant that SSc patients suffer from different clinical presentation of the plaque induced inflammatory periodontal disease.

Using capillaroscopy, Scardina et al. ${ }^{17}$ examined periodontal mucosa microcirculation in SSc patients. They reported an increased capillary caliber and a reduced number of visible capillary loops in SSc. A recent study of gingival biopsies by Ozceilk and co-workers ${ }^{21}$ showed that SSc samples had an increased level of gingival infiltration and an increased microvessel density but reduced levels of VEGF-A and VEGF$\mathrm{C}$ proteins expression, suggesting vessel degeneration. Findings from both studies are consistent with the current observations of higher BOP\% in the SSc group than in the controls. Nevertheless, the reasons why SSc subjects had deeper full-mouth mean PPD while the mean full-mouth PALs of both test and control groups remained comparable are yet to be elucidated. 
Similar to previous studies, ${ }^{13,14,16,22}$ this study found a widening of PDL space in SSc patients. SSc is an autoimmune disease of collagen-vascular disorder characterized by increased collagen production and tissue fibrosis. Alexandridis and White ${ }^{16}$ reported overall mean PDL space width of $0.292 \mathrm{~mm}$ in progressive systemic sclerosis subjects whereas their controls had overall mean of $0.220 \mathrm{~mm}$. Both our SSc as well as control groups appeared to have a larger PDL space of $0.357 \mathrm{~mm}$ or $0.331 \mathrm{~mm}$, respectively. The different between the previous and current study perhaps is due to the different radiological protocol of image acquisition. Further investigations are needed to clarify the differences in PDL width measurements. However, taking into consideration of limitations of both studies, the measured PDL space dimensions could be considered as similar and the differences measured between test and control group could be considered genuine. Auluck, ${ }^{23}$ Haers and Sailer $^{24}$ hypothesized the increased collagen production leads to hypertrophy of masticatory muscles. This produces excessive biting force and causes occlusal trauma to teeth, resulting in the widening of the periodontal ligament space in the absence of palpable periodontal disease. Rout et al. ${ }^{13}$ reported about one third of SSc people have the widening of the periodontal ligament space because hypertrophy of masticatory muscles does not occur in all patients. However, Mehra ${ }^{25}$ did not agree such hypothesis. He suggested the increased fibrosis of masticatory muscles and associated blood vessels of SSc people can cause ischemia and atrophy of masticatory muscles, which can reduce but not increase the 
biting forces. In addition, signs of occlusal trauma, such as angular bone defect and mobility of teeth, were not seen in patients with systemic sclerosis. Gonzale and Coleman $^{26}$ suggested increased collagen synthesis in the periodontal ligament leads to increase in the bulk of the ligament. This is accommodated at the expense of alveolar bone, causing an increase in the width of the periodontal ligament space. Further investigations are needed to identify the underlying reason of such radiographic observations.

\section{CONCLUSION}

This cross-sectional study adds to the observation that Hong Kong Chinese SSc patients, despite exhibiting similar levels of inadequate plaque control, mean full-mouth PAL, and number of standing teeth to non-SSc controls, they experienced a higher level of gingival inflammation, deeper mean full-mouth PPD, and widened radiographic PDL space. Such altered oral features, although conceivably attributable to gingival connective tissue and vascular changes in the SSc state, require continued investigation to elucidate the true biology behind the oral effects of this autoimmune disorder. 


\section{ACKNOWLEDGMENTS}

We would like to thank Trevor Lane, Knowledge Transfer Unit, Dental Faculty, the University of Hong Kong for editorial assistance. The work described in this paper was substantially supported by The University of Hong Kong Research and Conference Grant 200807176127 and partially supported by a grant from the Research Grants Council of the Hong Kong Special Administrative Region, China (772110M). 


\section{REFERENCES}

1. Vagra J. Systemic sclerosis B. Epidemiology, pathology, and pathogenesis. In: Klippel JH, Stone JH, Crofford LJ, White PH, eds. Primer on the Rheumatic Diseases, $13^{\text {th }}$ edn. New York: Springer; 2008: 351-358.

2. Walker UA, Tyndall A, Czirják L, et al. EUSTAR co-authors. Clinical risk assessment of organ manifestations in systemic sclerosis: a report from the EULAR Scleroderma Trials and Research group database. Ann Rheum Dis 2007:66:754-763.

3. Chifflot H, Fautrel B, Sordet C, Chatelus E, Sibilia J. Incidence and prevalence of systemic sclerosis: A systematic literature review. Semin Arthritis Rheum 2008:37:223-235.

4. Hinchcliff M, Varga J. Systemic sclerosis/scleroderma: A treatable multisystem disease. Am Fam Physician 2008:78:961-968.

5. Scully C, Cawson R.A. Medical Problems in Dentistry. $5^{\text {th }}$ edn. New York: Churchill Livingstone; 2005: 206-207.

6. Avouac J, Sordet C, Depinay C, et al. Systemic sclerosis-associated Sjögren's syndrome and relationship to the limited cutaneous subtype: results of a prospective study of sicca syndrome in 133 consecutive patients. Arthritis Rheum 2006:54:22432249. 
7. Bournia VK, Vlachoyiannopoulos PG, Selmi C, Moutsopoulos HM, Gershwin ME. Recent advances in the treatment of systemic sclerosis. Clin Rev Allergy Immunol 2009:36:176-200. Review.

8. Lin AT, Clements PJ, Furst DE. Update on disease-modifying antirheumatic drugs in the treatment of Systemic Sclerosis. Rheum Dis Clin North Am 2003:29:409-426.

9. Fischer, D.J., Patton, L.L. Scleroderma: oral manifestations and treatment challenges. Spec Care Dentist 2000:20:240-244.

10. Eversole LR, Jacobsen PL, Stone CE. Oral and gingival changes in systemic sclerosis (scleroderma). J Periodontol 1984:55:175-178.

11. Nagy G, Kovács J, Zeher M, Czirják L. Analysis of the oral manifestations of systemic sclerosis. Oral Surg Oral Med Oral Pathol 1994:77:141-146.

12. Hopper FE, Giles AD. Orofacial changes in systemic sclerosis-report of a case of resorption of mandibular angles and zygomatic arches. Br J Oral Surg 1982:20:129134.

13. Rout PG, Hamburger J, Potts AJ. Orofacial radiological manifestations of systemic sclerosis. Dentomaxillofac Radiol 1996:25:193-196.

14. Stafne EC, Austin LT. A characteristic dental finding in acrosclerosis and diffuse scleroderma. Am J Orthod Oral Surg 1944:30:C25-C29. 
15. Marmary Y, Glaiss R, Pisanty S. Scleroderma: oral manifestations. Oral Surg Oral Med Oral Pathol 1981:52:32-37.

16. Alexandridis C, White SC. Periodontal ligament changes in patients with progressive systemic sclerosis. Oral Surg Oral Med Oral Pathol 1984:58:113-118.

17. Scardina GA, Pizzigatti ME, Messina P. Periodontal microcirculatory abnormalities in patients with systemic sclerosis. J Periodontol 2005:76:1991-1995.

18. LeRoy EC, Black C, Fleischmajer R, et al. Scleroderma (systemic sclerosis): classification, subsets and pathogenesis. J Rheumatol 1988:15:202-205.

19. Cheng RHW, Leung WK, Corbet EF, King NM. Oral health status of adults with Down syndrome in Hong Kong. Spec Care Dentist 2007:27:134-138.

20. Genco RJ, Ho AW, Grossi SG, Dunford RG, Tedesco LA. Relationship of stress distress and inadequate coping behaviors to periodontal disease. J Periodontol 1999:35:1511-1520.

21. Ozcelik O, Haytac MC, Ergin M, Antmen B, Seydaoglu G. The immunohistochemical analysis of vascular endothelial growth factors A and C and microvessel density in gingival tissues of systemic sclerosis patients: their possible effects on gingival inflammation. Oral Surg Oral Med Oral Pathol Oral Radiol Endod 2008:105:481-485.

22. Wood RE, Lee P. Analysis of the oral manifestations of systemic sclerosis (scleroderma). Oral Surg Oral Med Oral Pathol 1988:65:172-178. 
23. Auluck A. Widening of periodontal ligament space and mandibular resorption in patients with systemic sclerosis. Dentomaxillofac Radiol 2007:36:441-442.

24. Haers PE, Sailer HF. Mandibular resorption due to systemic sclerosis. Case report of surgical correction of a secondary open bite deformity. Int J Oral Maxillofac Surg 1995:24:261-267. Review.

25. Mehra A. Periodontal space widening in patients with systemic sclerosis: a probable explanation. Dentomaxillofac Radiol 2008:37:183.

26. Gonzale T, Coleman GC. Periodontal manifestations of collagen vascular disorders. Periodontol 2000 1999:21:94-105.

Correspondence: Prof. W. K. Leung, Room 1B25, 34 Hospital Road, Prince Philip Dental Hospital, Faculty of Dentistry, The University of Hong Kong, Hong Kong SAR, China; Telephone: +852-28590417; Fax: 852-2858-2532, e-mail: ewkleung@hkucc.hku.hk. 
Table 1.

Characteristics of study participants

\begin{tabular}{|c|c|c|c|c|c|c|}
\hline \multirow{2}{*}{ Characteristic } & \multirow{2}{*}{ Categories } & \multicolumn{2}{|c|}{ Controls } & \multicolumn{2}{|c|}{ Cases (SSc) } & \multirow[b]{2}{*}{ p-value ${ }^{a}$} \\
\hline & & $\mathrm{n}$ & $(\%)$ & $\mathrm{n}$ & $(\%)$ & \\
\hline \multirow[t]{3}{*}{ Marital status } & Never married & 12 & (33.3) & 10 & $(27.8)$ & 0.765 \\
\hline & Married & 23 & $(63.9)$ & 24 & $(66.7)$ & \\
\hline & Separated/Divorced/Widowed & 1 & $(2.8)$ & 2 & $(5.6)$ & \\
\hline \multirow[t]{4}{*}{ Education } & Non/pre-school & 3 & $(8.3)$ & 3 & $(8.3)$ & 0.621 \\
\hline & Primary & 11 & $(30.6)$ & 12 & $(33.3)$ & \\
\hline & Secondary & 20 & $(55.6)$ & 16 & $(44.4)$ & \\
\hline & Tertiary or above & 2 & $(5.6)$ & 5 & $(13.9)$ & \\
\hline \multirow[t]{3}{*}{ Occupation } & Working/studying & 26 & $(58.3)$ & 15 & $(41.7)$ & 0.027 \\
\hline & Housewife & 5 & $(13.9)$ & 13 & $(36.1)$ & \\
\hline & Unemployed/retired & 5 & $(13.9)$ & 8 & $(22.2)$ & \\
\hline Monthly family & $\leq 9,999$ & 14 & $(38.9)$ & 9 & $(25.0)$ & 0.411 \\
\hline income & $10,000-24,999$ & 11 & $(30.6)$ & 10 & $(27.8)$ & \\
\hline \multirow[t]{2}{*}{$(\mathrm{HK} \$)^{\mathrm{b}}$} & $\geq 25,000$ & 6 & $(16.7)$ & 7 & $(19.4)$ & \\
\hline & Refused to disclose & 5 & (13.9) & 10 & $(27.8)$ & \\
\hline \multirow[t]{4}{*}{ Last dental visit } & $\leq 1$ year for check-up & 13 & $(36.1)$ & 10 & $(27.8)$ & 0.550 \\
\hline & $\leq 1$ year for dental problem & 6 & $(16.7)$ & 9 & $(25.0)$ & \\
\hline & $1-3$ years & 8 & $(22.2)$ & 5 & $(13.9)$ & \\
\hline & $>3$ years or never visited dentist & 9 & $(25.0)$ & 12 & (33.3) & \\
\hline \multirow[t]{3}{*}{ Brushing } & $\geq 2$ times daily & 24 & $(66.7)$ & 28 & $(77.8)$ & 0.565 \\
\hline & 1 time daily & 7 & (19.4) & 5 & (13.9) & \\
\hline & Occasionally & 5 & (13.9) & 3 & $(8.3)$ & \\
\hline \multirow[t]{3}{*}{ Flossing } & Every day & 4 & (11.1) & 12 & (33.3) & 0.076 \\
\hline & Occasionally & 9 & $(25.0)$ & 7 & $(19.4)$ & \\
\hline & Never & 23 & $(63.9)$ & 17 & $(47.2)$ & \\
\hline
\end{tabular}


${ }^{\mathrm{a}}$ Chi-square test
${ }^{\mathrm{b}}$ USD $\$ 1.00=$ HKD $\$ 7.80$

6

7

8

10

11

12

13

14

15

16

17

18

19

20

21

22

23

24

25

26

27

28

29

30

31

32

33

34

35

36

37

38

39

40

41

42

43

44

45

46

47

48

49

50

51

52

53

54

55

56

57

58

59 
Table 2.

Clinical characteristics of study participants

\begin{tabular}{|c|c|c|c|c|c|c|c|c|c|c|}
\hline \multirow[b]{2}{*}{ Characteristic } & \multicolumn{3}{|c|}{ Controls } & \multicolumn{3}{|c|}{ Cases (SSc) } & \multicolumn{3}{|c|}{$95 \% \mathrm{CI}$} & \multirow[b]{2}{*}{$\mathrm{p}$-value ${ }^{\mathrm{a}}$} \\
\hline & (Mean & \pm & SD) & (Mean & \pm & SD) & Diff & Lower & Upper & \\
\hline Erupted teeth (n) & 23.7 & \pm & 6.0 & 24.9 & \pm & 5.2 & 1.2 & -0.46 & 2.86 & 0.151 \\
\hline $\operatorname{Pl}(\%)$ & 65.0 & \pm & 12.5 & 69.3 & \pm & 18.0 & 4.3 & -1.11 & 9.71 & 0.116 \\
\hline $\mathrm{BOP}(\%)$ & 49.3 & \pm & 22.6 & 78.4 & \pm & 19.6 & 29.1 & 26.94 & 31.27 & $<0.001$ \\
\hline Mean full mouth $\operatorname{PPD}(\mathrm{mm})$ & 1.92 & \pm & 0.44 & 2.52 & \pm & 0.58 & 0.6 & 0.43 & 0.77 & $<0.001$ \\
\hline$\%$ sites $\mathrm{PPD}=4-5 \mathrm{~mm}$ & 4.77 & \pm & 4.80 & 12.36 & \pm & 8.24 & 7.59 & 7.15 & 8.03 & $<0.001$ \\
\hline$\%$ sites $\mathrm{PPD} \geq 6 \mathrm{~mm}$ & 0.76 & \pm & 1.41 & 1.34 & \pm & 2.23 & 0.58 & -0.13 & 1.29 & 0.106 \\
\hline Mean full mouth PAL(mm) & 3.17 & \pm & 1.29 & 3.19 & \pm & 0.94 & 0.02 & -0.18 & 0.22 & 0.843 \\
\hline
\end{tabular}


Table 3.

Radiographic periodontal ligament (PDL) width (mm)

\begin{tabular}{|c|c|c|c|c|c|c|c|c|c|c|c|c|}
\hline \multirow[b]{2}{*}{ Teeth } & \multicolumn{4}{|c|}{ Controls } & \multicolumn{4}{|c|}{ Cases (SSc) } & \multicolumn{3}{|c|}{$95 \% \mathrm{CI}$} & \multirow[b]{2}{*}{ p-value ${ }^{b}$} \\
\hline & No. ${ }^{a}$ & Mean & \pm & SD & No. ${ }^{a}$ & Mean & \pm & SD & Diff & Lower & Upper & \\
\hline \multicolumn{13}{|l|}{ Anterior } \\
\hline Maxillary central incisor & 36 & 0.315 & \pm & 0.062 & 33 & 0.373 & \pm & 0.090 & 0.057 & 0.014 & 0.100 & 0.010 \\
\hline Mandibular lateral incisor & 36 & 0.309 & \pm & 0.075 & 34 & 0.324 & \pm & 0.073 & 0.014 & -0.025 & 0.053 & 0.467 \\
\hline Mandibular canine & 36 & 0.327 & \pm & 0.044 & 36 & 0.343 & \pm & 0.070 & 0.015 & -0.015 & 0.045 & 0.302 \\
\hline Overall (Anterior) & 108 & 0.317 & \pm & 0.039 & 103 & 0.346 & \pm & 0.060 & 0.029 & 0.002 & 0.056 & 0.038 \\
\hline
\end{tabular}

\section{Posterior}

Maxillary second premolar

$\begin{array}{rrrrrrrrrrrr}31 & 0.331 & \pm & 0.080 & 32 & 0.353 & \pm & 0.086 & 0.022 & -0.020 & 0.064 & 0.303 \\ 32 & 0.343 & \pm & 0.070 & 34 & 0.391 & \pm & 0.109 & 0.049 & 0.010 & 0.088 & 0.015 \\ 35 & 0.337 & \pm & 0.072 & 35 & 0.338 & \pm & 0.076 & 0.001 & -0.032 & 0.034 & 0.952 \\ 34 & 0.346 & \pm & 0.060 & 32 & 0.403 & \pm & 0.081 & 0.057 & 0.031 & 0.074 & <0.001 \\ 132 & 0.339 & \pm & 0.042 & 133 & 0.370 & \pm & 0.063 & 0.031 & 0.010 & 0.052 & 0.005\end{array}$

Maxillary teeth

$99 \quad 0.330 \pm 0.046$

99.372

\begin{tabular}{l}
$+076 \quad 0.043$ \\
\hline
\end{tabular}

$0.012 \quad 0.073$

0.008

Mandibular teeth

$\begin{array}{llllllllll}141 & 0.330 & \pm & 0.035 & 137 & 0.351 & \pm & 0.058 & 0.021\end{array}$

$0.001 \quad 0.041$

0.043

Overall

$240 \quad 0.330 \pm 0.032$

$2360.360 \quad \pm \quad 0.057 \quad 0.030$

$0.010 \quad 0.050$

0.005

${ }^{\mathrm{a}}$ No. $=$ number of teeth measured

${ }^{\mathrm{b}}$ Paired t-test 